\title{
VERGLEICH DER LEHRPLANMATRIX DES UNDERGRADUATE CHEMISTRY COURSE AM IFAP MIT DEM INHALT VON ENADE.
}

\section{ORIGINALER ARTIKEL}

SANTOS, Lívia Maria Sousa dos ${ }^{1}$, TATY, Salvador Rodrigues², FERNANDES, Erlyson Farias $^{3}$, FECURY, Amanda Alves ${ }^{4}$, DENDASCK, Carla Viana ${ }^{5}$, OLIVEIRA, Euzébio $\mathrm{de}^{6}$, DIAS, Claudio Alberto Gellis de Mattos $^{7}$

SANTOS, Lívia Maria Sousa dos. Et al. Vergleich der lehrplanmatrix des undergraduate chemistry course am IFAP mit dem inhalt von ENADE. Revista Científica Multidisciplinar Núcleo do Conhecimento. Jahrgang 06, Ed. 03, Vol. 14, S. 29-40. März 2021. ISSN: 2448-0959, Zugangslink: https://www.nucleodoconhecimento.com.br/bildung-de/lehrplanmatrix, $\quad$ DOI: 10.32749/nucleodoconhecimento.com.br/bildung-de/lehrplanmatrix

\section{ZUSAMMENFASSUNG}

Curricular Matrix ist der Satz von Curriculumkomponenten (Disziplinen), die den Inhalt der Bildung eines Studenten in einem Kurs garantieren und ständigen Veränderungen aufgrund der Geschwindigkeit, mit der akademisch-technologische Innovationen

\footnotetext{
${ }^{1}$ Student des Technischen Kurses für Chemie (zweiter Grad) des Instituts für Grund-, Technik- und Technologiepädagogik (IFAP).

${ }^{2}$ Chemiker, Master in Chemie (UFMA), Professor und Forscher des Lizentiatskurses in Chemie am Institut für grundlegende, technische und technologische Bildung von Amapá (IFAP), Koordinator des technischen Studiengangs in Chemie (IFAP).

${ }^{3}$ Chemiker, Master in Chemie (UFPA), Professor und Forscher des Lizentiats in Chemie am Institut für grundlegende, technische und technologische Bildung von Amapá (IFAP).

${ }^{4}$ Biomedizin, PhD in Tropenkrankheiten, Professor und Forscher des Medizinkurses am Campus Macapá, Bundesuniversität Amapá (UNIFAP).

${ }^{5}$ Theologe, PhD in Klinischer Psychoanalyse. Er arbeitet seit 15 Jahren mit wissenschaftlicher Methodik (Forschungsmethode) in der wissenschaftlichen Produktionsberatung für Master- und Doktoranden. Spezialist für Marktforschung und Gesundheitsforschung.

${ }^{6}$ Biologe, PhD in Tropenkrankheiten, Professor und Forscher des Sportunterrichts an der Föderalen Universität von Pará (UFPA).

${ }^{7}$ Biologe, Doktor in Verhaltenstheorie und Verhaltensforschung, Professor und Forscher des Lizentiatskurses in Chemie am Institut für grundlegende, technische und technologische Bildung von Amapá (IFAP) und des Graduiertenprogramms in beruflicher und technologischer Bildung (PROFEPT IFAP).

RC: 80986

Ссылка доступа: https://www.nucleodoconhecimento.com.br/образование-ru/матрицы-
} 
präsentiert werden. Der höhere Kurs in Chemie, der vom Bundesinstitut für Amapá aangeboten angeboten wird, basiert auf seinen Zielen angesichts der Berufsausbildung des Studenten in der Hochschule. Ziel dieser Arbeit war es, den Chemiegehalt der National Student Performance Exam (ENADE) mit der Curriculummatrix des Bachelor-Abschlusses in Chemie des Bundesinstituts für Amapá (IFAP) in den Jahren 2011, 2014 und 2017 zu vergleichen. Die Bewertung von ENADE für Chemie-Absolventen erfordert, dass der ausgebildete Fachmann wie erwartet über Kenntnisse der spezifischen Inhalte verfügt. Aber sie sucht in ihnen ein Wissen, das die Dynamik eines Klassenzimmers umfasst. Auf dieser Grundlage stellen wir eine Vorliebe für bestimmte und unspezifische Fächer fest, die Informationen über die praktische Ausbildung der Studierenden sucht. Der Studiengang Chemie am IFAP scheint mehr als genug Arbeitsbelastung zu haben, damit sich der Student an der ENADE gut vorbereiten und gut abschneidet.

Schlagworte: Curriculum Matrix, ENADE, Bachelor es degree, Chemistry.

\section{EINLEITUNG}

Curricular Matrix ist der Satz von Curricular Komponenten (Disziplinen), die den Inhalt der Bildung eines Studenten in einem Kurs garantiert. Diese Komponenten decken obligatorische und nicht obligatorische Materialien ab. Unterstützt durch den PDI (Institutional Development Plan) und das PPC (Pedagogical Course Project) strukturieren das Curriculum auf der Grundlage von Lehr- und außerschulischen Praktika, praktischen Laborkursen (falls vorhanden), Überwachung, Kursabschlussarbeit, ergänzenden Aktivitäten und dem gewünschten Profil des Schülers, der den Kurs bereits abgeschlossen hat und sich auf dem Arbeitsmarkt befindet (egresso) (Carneiro et al., 2017).

Die Curriculum-Matrix durchläuft ständige Veränderungen aufgrund der Geschwindigkeit, mit der akademische und technologische Innovationen präsentiert werden, was die Notwendigkeit von Änderungen im Curriculum bringt, um mehr Lernen

RC: 80986

Ссылка доступа: https://www.nucleodoconhecimento.com.br/образование-ru/матрицы- 
im Kurs zu gewährleisten, um sicherzustellen, dass die Studenten besser qualifiziert und fit für den Arbeitsmarkt sein können (Carneiro et al., 2017).

Der höhere Kurs in Chemie, der vom Bundesinstitut für Amapá aangeboten angeboten wird, basiert auf seinen Zielen angesichts der Berufsausbildung des Studenten in der Hochschule. Technologische und wissenschaftliche Erkenntnisse werden zusammen mit den theoretischen und praktischen Grundlagen in allen spezifischen Bereichen des Chemiebereichs zur Verfügung gestellt, die zur Ausbildung eines qualifizierten Fachmanns beitragen, um so in Zukunft als Chemielehrer in der Grundbildung tätig zu werden, mit einem stärkeren Schwerpunkt auf dem Gymnasium (Ifap, 2011).

Die National Student Performance Exam (ENADE) ist eine Prüfung, die am Ende der Hochschulbildung angewendet wird, um das Wissen der Absolventen jedes Kurses zu messen. Dies wird in zwei Teile unterteilt, den allgemeinen, der von allen Kursen eines bestimmten Jahres durchgeführt wird, um kognitive Fähigkeiten wie Interpretation von Graphen, logisches Denken und andere zu messen; und den spezifischen Teil für jeden Kurs, wobei das jeweilige Wissen gemessen wird. (Wainer und Melguizo, 2018). Die Prüfung wird alle drei Jahre in einem Zyklus durchgeführt und ihre Anwendung ist obligatorisch (Lima et al., 2019).

Jeder, der fünfundsiebzig Prozent oder mehr der Mindestkursbelastung absolviert hat, darf den Test gemäß den Bestimmungen für Absolventen von Hochschultechnologiekursen ablegen. Die Einschreibung dieses Teilnehmers muss von der Hochschule (IES) gemäß $§ 6$ der Kunst erfolgen. 5 des Gesetzes 10.861 / 2004 Der registrierte Teilnehmer muss am Testort teilnehmen. Seine Abwesenheit führt ohne plausible Begründung zu Unregelmäßigkeiten in der Schulaufzeichnung sowie zur Ausstellung des Abschlussdiploms des Kurses (Enade, 2020).

RC: 80986

Ссылка доступа: https://www.nucleodoconhecimento.com.br/образование-ru/матрицы- 


\section{ZIELE}

Vergleichen Sie den Chemiegehalt der National Student Performance Exam (ENADE) mit der Curriculummatrix des Chemie-Hochschulkurses des Bundesinstituts für Amapá (IFAP) in den Jahren 2011, 2014 und 2017.

\section{MATERIAL UND METHODEN}

Die Forschung wurde mit Hilfe des Google-Suchtools (http://inep.gov.br/educacaosuperior/enade/provas-e-gabaritos) (Inep, 2020) aus dem weltweiten Computernetzwerk durchgeführt. Die Disziplin Chemie-Abschluss wurde ausgewählt und die Jahre 20112014 und 2017, mit der Box (mit dem Jahr) auf der Website zur Verfügung. Anschließend wurde ein Vergleich zwischen diesen und dem programmatischen Inhalt des Studiums der Chemie am Instituto Federal do Amapá, IFAP (https://www.ifap.edu.br/) (Ifap, 2011) angestellt. Die bibliographische Forschung wurde in wissenschaftlichen Artikeln, im Home Office durchgeführt. Die Daten wurden in die Excel-Anwendung kompiliert, eine Komponente der Microsoft Corporation Office-Suite.

\section{ERGEBNISSE}

Die ENADE-Chemieausgaben 2011, 2014 und 2017 nach Inhaltstyp jeder Frage sind in Abbildung 1 dargestellt. Fragen, die nur eine Art von Inhalt (einfacher Inhalt) enthalten, stellen 30 der 90 Testfragen der drei Jahre dar (33,33\%). Die Fragen, die mehr als einen Inhalt haben (überlappende Inhalte), sind 60 der 90 Fragen $(66,67 \%)$.

RC: 80986

Ссылка доступа: https://www.nucleodoconhecimento.com.br/образование-ru/матрицы- 
Abbildung 1 - zeigt die Anzahl der ENADE-Chemieprobleme aus den Jahren 2011, 2014 und 2017 nach Inhaltstyp jeder Frage.

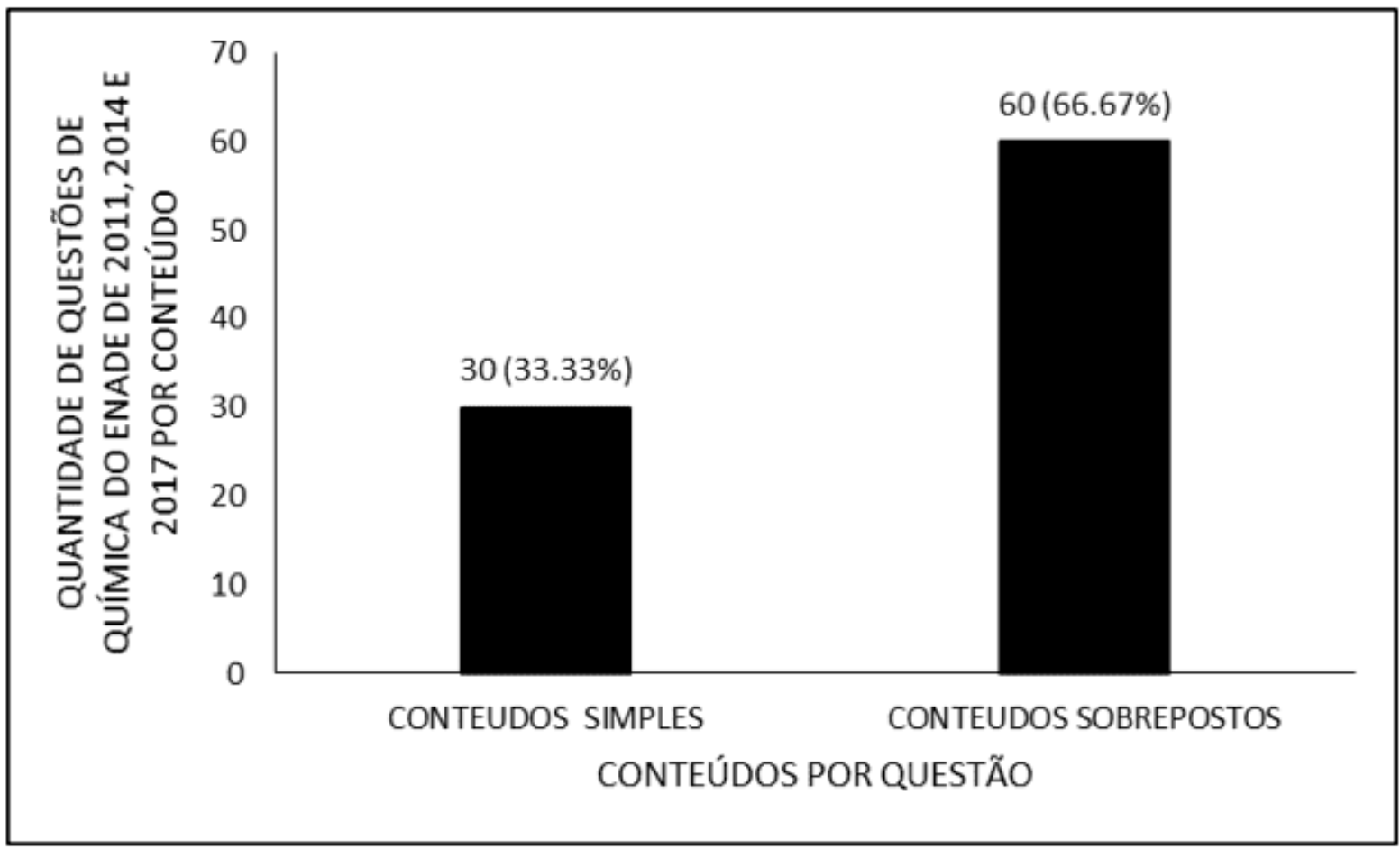

Tabelle 1 zeigt die Verteilung (Menge und Prozentsatz) der Fragen in den Inhalten (einfach und überlappend) in der ENADE 2011, 2014 und 2017 nach Frage. Unter den Fragen mit einfachem Inhalt wurden in den Bewertungen dieser Jahre 10 Inhalte nicht berechnet (1,11\%); fünf Inhalte erschienen einmal (2,22\%); in Inhalt (Organische Verbindungen: Reaktionen und Mechanismen; natürliche und synthetische Makromoleküle) wurde in drei Fragen (3,33\%) berechnet; und eine andere (Öffentliche Politik und inre Auswirkungen auf den Chemieunterricht) wurde siebenmal $(7,78 \%)$ berechnet. $\mathrm{Zu}$ den am präsentesten Inhalten gehörten in drei Fragen $(3,33 \%)$ "Curricular Projekte und Vorschläge im Chemie- und Evaluierungsunterricht und in ihren Beziehungen zu den verschiedenen Konzepten des Lehrens und Lernens + Didaktische Ressourcen für den Chemieunterricht". Die anderen Kombinationen lernen in jeder Frage eine.

RC: 80986

Ссылка доступа: https://www.nucleodoconhecimento.com.br/образование-ru/матрицыбакалавриата 
Tabelle 1 - zeigt die Verteilung (Menge und Prozentsatz) der Fragen im Inhalt (einfach und überlappend) in der ENADE 2011, 2014 und 2017, nach Frage.

\begin{tabular}{|c|c|c|}
\hline CONTEÚDOS SIMPLES & $\begin{array}{l}\text { Quantidade } \\
\text { de questőes }\end{array}$ & $\begin{array}{l}\text { Porcentagem } \\
\text { de questőes }\end{array}$ \\
\hline Elementos químicos (química in orgânica) & 0 & $0.00 \%$ \\
\hline Estrutura molecular (química in orgânica) & 0 & $0.00 \%$ \\
\hline Estudo de substâncias e transformações químicas (físico-quimica) & 0 & $0.00 \%$ \\
\hline Métodos de análise em química: caracterização e quantificação & 0 & $0.00 \%$ \\
\hline Gases (química geral) & 0 & $0.00 \%$ \\
\hline Compostos inorgânicos de elementos representativos e de coordenação & 0 & $0.00 \%$ \\
\hline Bioquímica: estrutura de biomoléculas, biossíntese e metabolismo & 0 & $0.00 \%$ \\
\hline $\begin{array}{c}\text { Estratégias de ensino e de avaliação em Química e suas relações com as diferentes concepções de } \\
\text { ensino e aprendizagem }\end{array}$ & 0 & $0.00 \%$ \\
\hline Estrutura molecular e de sólidos iônicos e metálicos & 0 & $0.00 \%$ \\
\hline Compostos orgânicos + bioquímica & 0 & $0.00 \%$ \\
\hline Estrutura atômica (química geral) & 1 & $1.11 \%$ \\
\hline Química ambiental & 1 & $1.11 \%$ \\
\hline $\begin{array}{l}\text { A história da Química no contexto do desenvolvimento científico e tecnológico e a sua relação com o } \\
\text { ensino de Química }\end{array}$ & 1 & $1.11 \%$ \\
\hline Recursos didáticos para o ensino de Química & 1 & $1.11 \%$ \\
\hline Relações entre ciência, tecnologia, sociedade e ambiente no ensino de Química & 1 & $1.11 \%$ \\
\hline Estados dispersos: soluções e sistemas coloidais & 2 & $2.22 \%$ \\
\hline Termodinâmica (química geral) & 2 & $2.22 \%$ \\
\hline cinética química (química geral) & 2 & $2.22 \%$ \\
\hline $\begin{array}{c}\text { Normas de segurança e operações de laboratório utilizadas em síntese, purificação, caracterização e } \\
\text { quantificação de substâncias e em determinações físico-químicas }\end{array}$ & 2 & $2.22 \%$ \\
\hline Projetos e propostas curriculares no ensino de Química & 2 & $2.22 \%$ \\
\hline A experimentação no ensino de Química & 2 & $2.22 \%$ \\
\hline Eletroquímica (química geral) & 3 & $3.33 \%$ \\
\hline Compostos orgânicos: reações e mecanismos; macromoléculas naturais e sintéticas & 3 & $3.33 \%$ \\
\hline As políticas públicas e suas implicaçôes para o ensino de Química & 7 & $7.78 \%$ \\
\hline TOTAL & 30 & $33.33 \%$ \\
\hline
\end{tabular}

RC: 80986

Ссылка доступа: https://www.nucleodoconhecimento.com.br/образование-ru/матрицы- 
CONTEÚDOS SOBREPOSTOS

Quantidade Porcentagem

\begin{tabular}{|c|c|c|}
\hline CONTEÚDOS SOBREPOSTOS & $\begin{array}{l}\text { Quantidade } \\
\text { de questōes }\end{array}$ & $\begin{array}{l}\text { Porcentagem } \\
\text { de questōes }\end{array}$ \\
\hline $\begin{array}{l}\text { Métodos de análise em química: caracterização e quantificação + Compostos orgânicos: reaçōes e } \\
\text { mecanismos; macromoléculas naturais e sintéticas + Bioquímica: estrutura de biomoléculas, } \\
\text { biossíntese e metabolismo + Química ambiental }\end{array}$ & 1 & $1.11 \%$ \\
\hline $\begin{array}{l}\text { Elementos químicos (química inorgânica) + Estrutura atômica (química geral) + Estrutura molecular } \\
\text { (química inongânica) + Compostos inorgânicos de elementos representativos e de coordenação + } \\
\text { Compostos orgânicos: reaçōes e mecanismos; macromoléculas naturais e sintéticas }\end{array}$ & 1 & $1.11 \%$ \\
\hline $\begin{array}{l}\text { Elementos químicos (química inorgânica) + Estudo de substâncias e transformaçōes químicas (físico- } \\
\text { química) + Gases (química geral) + Eletroquímica (química geral) }\end{array}$ & 1 & $1.11 \%$ \\
\hline $\begin{array}{l}\text { Estrutura atômica (química geral) + Estrutura molecular (química inorgânica) + Compostos inongânicos } \\
\text { de elementos representativos e de coordenação + Estrutura molecular e de sólidos iônicos e metálicos } \\
\text { + Elementos químicos (química inorgânica) }\end{array}$ & 1 & $1.11 \%$ \\
\hline $\begin{array}{c}\text { Elementos químicos (química inorgânica) + Estrutura atômica (química geral) + Eletroquímica (química } \\
\text { geral) + A história da Química no contexto do desenvolvimento científico e tecnológico e a sua relaçăo } \\
\text { como ensino de Química + Estrutura molecular e de sólidos iônicos e metálicos }\end{array}$ & 1 & $1.11 \%$ \\
\hline $\begin{array}{l}\text { Compostos orgânicos: reações e mecanismos; macromoléculas + Bioquímica: estrutura de } \\
\text { biomoléculas, biossíntese e metabolismo + Compostos orgânicos + bioquímica }\end{array}$ & 1 & $1.11 \%$ \\
\hline $\begin{array}{l}\text { Compostos ongânicos: reações e mecanismos; macromoléculas naturais e sintéticas + Bioquímica: } \\
\text { estrutura de biomoléculas, biossíntese e metabolismo + Compostos orgânicos + bioquímica }\end{array}$ & 1 & $1.11 \%$ \\
\hline $\begin{array}{l}\text { Elementos químicos (química inorgânica) +Estrutura molecular (química inorgânica) + Métodos de } \\
\text { análise em química: caracterização e quantificação + Compostos inorgânicos de elementos } \\
\text { representativos e de coordenaçăo + Estrutura molecular e de sólidos iônicos e metálicos }\end{array}$ & 1 & $1.11 \%$ \\
\hline $\begin{array}{l}\text { Métodos de análise em química: caracterização e quantificação + Química ambiental + Estudo de } \\
\text { substâncias e transformaçóes químicas (físico-química) }\end{array}$ & 1 & $1.11 \%$ \\
\hline $\begin{array}{c}\text { Estudo de substâncias e transformaçōes químicas (físico-química) + cinética química (química geral) + } \\
\text { Eletroquímica (química geral) + Compostos orgânicos: reaçōes e mecanismos; macromoléculas naturais } \\
\text { e sintéticas + Bioquímica: estrutura de biomoléculas, biossíntese e metabolismo + Compostos } \\
\text { orgânicos + bioquímica }\end{array}$ & 1 & $1.11 \%$ \\
\hline $\begin{array}{l}\text { Elementos químicos (química inorgânica) + Estudo de substâncias e transformações químicas (físico- } \\
\text { química) + Métodos de análise em química: caracterização e quantificação + Química ambiental + A } \\
\text { experimentação no ensino de Química }\end{array}$ & 1 & $1.11 \%$ \\
\hline $\begin{array}{c}\text { Projetos e propostas curriculares no ensino de Química + As políticas públicas e suas implicações para o } \\
\text { ensino de Química }\end{array}$ & 1 & $1.11 \%$ \\
\hline $\begin{array}{c}\text { Projetos e propostas curriculares no ensino de Química + Estratégias de ensino e de avaliaçāo em } \\
\text { Química e suas relaçōes com as diferentes concepçōes de ensino e aprendizagem + Recursos didáticos } \\
\text { para o ensino de Química }\end{array}$ & 1 & $1.11 \%$ \\
\hline $\begin{array}{l}\text { Projetos e propostas curriculares no ensino de Química + Recursos didáticos para o ensino de Química + } \\
\text { As políticas públicas e suas implicações para o ensino de Química }\end{array}$ & 1 & $1.11 \%$ \\
\hline $\begin{array}{l}\text { Projetos e propostas curriculares no ensino de Química + Relaçōes entre ciência, tecnologia, sociedade } \\
\text { e ambiente no ensino de Química + A experimentação no ensino de Química + As políticas públicas e } \\
\text { suas implicaçōes para o ensino de Química }\end{array}$ & 1 & $1.11 \%$ \\
\hline $\begin{array}{c}\text { Projetos e propostas curriculares no ensino de Química + As políticas públicas e suas implicações para o } \\
\text { ensino de Química }\end{array}$ & 1 & $1.11 \%$ \\
\hline
\end{tabular}

RC: 80986

Ссылка доступа: https://www.nucleodoconhecimento.com.br/образование-ru/матрицы- 


\begin{tabular}{|c|c|c|}
\hline CONTEÚDOS SOBREPOSTOS & $\begin{array}{l}\text { Quantidade } \\
\text { de questŏes }\end{array}$ & $\begin{array}{l}\text { Porcentagem } \\
\text { de questões }\end{array}$ \\
\hline $\begin{array}{l}\text { Estrutura atômica (química ge ral) + Eletroquímica (química geral) + A história da Química no contexto do } \\
\text { desenvolvimento científico e tecnológico e a sua relação com o ensino de Química + Relações entre } \\
\text { ciência, tecnologia, sociedade e ambiente no ensino de Química }\end{array}$ & 1 & $1.11 \%$ \\
\hline $\begin{array}{l}\text { Métodos de análise em química: caracterização e quantificação + Química ambiental + Relações entre } \\
\text { ciência, tecnologia, sociedade e ambiente no ensino de Química + As políticas públicas e suas } \\
\text { implicações para o ensino de Química }\end{array}$ & 1 & $1.11 \%$ \\
\hline $\begin{array}{l}\text { Elementos químicos (química inorgânica) + Estrutura atômica (química geral) + Estrutura molecular } \\
\text { (química inorgânica) + Métodos de análise em química: caracterização e quantificação + Compostos } \\
\text { orgânicos: reações e mecanismos; macromoléculas naturais e sintéticas + Bioquímica: estrutura de } \\
\text { biomoléculas, biossíntese e metabolismo + Compostos orgânicos + bioquímica }\end{array}$ & 1 & $1.11 \%$ \\
\hline $\begin{array}{l}\text { Elementos químicos (química inorgânica) + Estrutura atômica (química geral) + Estrutura molecular } \\
\text { (química inorgânica) + Métodos de análise em química: caracterização e quantificação + Compostos } \\
\text { inorgânicos de elementos representativos e de coordenação + Estratégias de ensino e de avaliação em } \\
\text { Química e suas relações com as dife rentes concepções de ensino e aprendizagem + Recursos didáticos } \\
\text { para o ensino de Química + A experimentação no ensino de Química }\end{array}$ & 1 & $1.11 \%$ \\
\hline $\begin{array}{c}\text { Estudo de substâncias e transformações químicas (físico-química) + Termodinâmica (química geral) + } \\
\text { Eletroquímica (química geral) }\end{array}$ & 1 & $1.11 \%$ \\
\hline $\begin{array}{l}\text { Métodos de análise em química: caracterização e quantificação + Estados dispersos: soluções e } \\
\qquad \text { sistemas coloidais + Química ambiental }\end{array}$ & 1 & $1.11 \%$ \\
\hline Estudo de substâncias e transformações químicas (físico-química) + Gases (química geral) & 1 & $1.11 \%$ \\
\hline $\begin{array}{c}\text { Elementos químicos (química inorgânica) + Estrutura molecular (química inorgânica) + Compostos } \\
\text { inorgânicos de elementos representativos e de coordenação + Estrutura molecular e de sólidos iônicos } \\
\text { e metálicos }\end{array}$ & 1 & $1.11 \%$ \\
\hline Estudo de substâncias e transformações químicas (físico-química) + Termodinâmica (química geral) & 1 & $1.11 \%$ \\
\hline $\begin{array}{l}\text { Estados dispersos: soluções e sistemas coloidais + Estratégias de ensino e de avaliação em Química e } \\
\text { suas relações com as diferentes concepções de ensino e aprendizagem + Recursos didáticos para o } \\
\text { ensino de Química + A experimentação no ensino de Química }\end{array}$ & 1 & $1.11 \%$ \\
\hline Métodos de análise em química: caracterização e quantificação + Química ambiental & 1 & $1.11 \%$ \\
\hline $\begin{array}{l}\text { Estrutura molecular (química inorgânica) + Métodos de análise em química: caracterização e } \\
\text { quantificação + Compostos orgânicos: reações e mecanismos; macromoléculas naturais e sintéticas }\end{array}$ & 1 & $1.11 \%$ \\
\hline $\begin{array}{l}\text { Estudo de substâncias e transformações químicas (físico-química) + Métodos de análise em química: } \\
\text { caracterização e quantificação + Química ambiental }\end{array}$ & 1 & $1.11 \%$ \\
\hline
\end{tabular}

RC: 80986

Ссылка доступа: https://www.nucleodoconhecimento.com.br/образование-ru/матрицы- 


\begin{tabular}{|c|c|c|}
\hline $\begin{array}{l}\text { Compostos orgânicos: reações e mecanismos; macromoléculas naturais e sintéticas + Bioquímica: } \\
\text { estrutura de biomoléculas, biossíntese e metabolismo + Compostos orgânicos + bioquímica }\end{array}$ & 1 & $1.11 \%$ \\
\hline $\begin{array}{l}\text { Estrutura molecular (química inorgânica) + Métodos de análise em química: caracterização e } \\
\text { quantificação + Compostos orgânicos: reações e mecanismos; macromoléculas naturais e sintéticas + } \\
\text { Bioquímica: estrutura de biomoléculas, biossíntese e metabolismo + Compostos orgânicos + bioquímica }\end{array}$ & 1 & $1.11 \%$ \\
\hline $\begin{array}{l}\text { Elementos químicos (química inorgânica) + estrutura atômica (química ge ral) + Estrutura molecular } \\
\text { (química inorgânica) + Termodinâmica (química geral) + Compostos inorgânicos de elementos } \\
\text { representativos e de coordenação + Compostos orgânicos: reações e mecanismos; macromoléculas } \\
\text { naturais e sintéticas + Bioquímica: estrutura de biomoléculas, biossíntese e metabolismo + Estrutura } \\
\text { molecular e de sólidos iônicos e metálicos + Compostos orgânicos + bioquímica }\end{array}$ & 1 & $1.11 \%$ \\
\hline $\begin{array}{c}\text { Termodinâmica (química geral) + Compostos orgânicos: reações e mecanismos; macromoléculas } \\
\text { naturais e sintéticas }\end{array}$ & 1 & $1.11 \%$ \\
\hline $\begin{array}{l}\text { Projetos e propostas curriculares no ensino de Química + as políticas públicas e suas implicações para o } \\
\text { ensino de Química }\end{array}$ & 1 & $1.11 \%$ \\
\hline $\begin{array}{l}\text { Estratégias de ensino e de avaliação em Química e suas relações com as dife rentes concepções de } \\
\text { ensino e aprendizagem + as políticas públicas e suas implicações para o ensino de Química }\end{array}$ & 1 & $1.11 \%$ \\
\hline $\begin{array}{l}\text { Projetos e propostas curriculares no ensino de Química + Estratégias de ensino e de avaliação em } \\
\text { Química e suas relações com as diferentes concepções de ensino e aprendizagem }\end{array}$ & 1 & $1.11 \%$ \\
\hline $\begin{array}{l}\text { Projetos e propostas curriculares no ensino de Química + Estratégias de ensino e de avaliação em } \\
\text { Química e suas relações com as diferentes concepções de ensino e aprendizagem + as políticas públicas } \\
\text { e suas implicações para o ensino de Química }\end{array}$ & 1 & $1.11 \%$ \\
\hline $\begin{array}{l}\text { Estudo de substâncias e transformações químicas (físico-química) + Métodos de análise em química: } \\
\text { caracte rização e quantificação + Eletroquímica (química geral) }\end{array}$ & 1 & $1.11 \%$ \\
\hline
\end{tabular}

RC: 80986

Ссылка доступа: https://www.nucleodoconhecimento.com.br/образование-ru/матрицы- 
Cinética química (química geral) + Bioquímica: estrutura de biomoléculas, biossíntese e metabolismo

Elementos químicos (química inorgânica) + Estrutura molecular (química inorgânica) + Gases (química geral) + Compostos inorgânicos de elementos representativos e de coordenação + Estrutura molecular e de sólidos iônicos e metálicos

\begin{tabular}{|c|c|c|}
\hline e de sólidos iônicos e metálicos & & \\
\hline $\begin{array}{l}\text { Estrutura molecular (química inorgânica) + Compostos orgâni cos: reações e mecanismos; } \\
\text { macromoléculas naturais e sintéticas + Compostos orgânicos + bioquímica }\end{array}$ & 1 & $1.11 \%$ \\
\hline $\begin{array}{c}\text { Elementos químicos (química inorgânica) + Estrutura atômica (química geral) + Estrutura mol ecular } \\
\text { (química inorgânica) + Eletroquímica (química geral) }\end{array}$ & 1 & $1.11 \%$ \\
\hline $\begin{array}{c}\text { Estrutura atômica (química geral) + Métodos de análise em química: caracterização e quantificação + } \\
\text { Compostos inorgâni cos de elementos representativos e de coordenaç̧ão + A experimentação no ensino } \\
\text { de Química }\end{array}$ & 1 & $1.11 \%$ \\
\hline $\begin{array}{c}\text { Métodos de análise em química: caracte rização e quantificação + A experimentação no ensino de } \\
\text { Química }\end{array}$ & 1 & $1.11 \%$ \\
\hline Termodinâmica (química geral) + Eletroquímica (química geral) & 1 & $1.11 \%$ \\
\hline $\begin{array}{l}\text { Estudo de substâncias e transformações químicas (físico-química) + Termodinâmica (química geral) + } \\
\text { cinética química (química geral) }\end{array}$ & 1 & $1.11 \%$ \\
\hline Cinética química (química geral) + Bioquímica: estrutura de biomoléculas, biossíntese e metabolismo & 1 & $1.11 \%$ \\
\hline Termodinâmica (química geral) + Gases (química geral) & 1 & $1.11 \%$ \\
\hline $\begin{array}{l}\text { Métodos de análise em química: caracterização e quantificação + Compostos orgânicos: reações e } \\
\text { mecanismos; macromoléculas naturais e sintéticas + Bioquímica: estrutura de biomoléculas, } \\
\text { biossíntese e metabolismo + Compostos orgânicos + bioquímica }\end{array}$ & 1 & $1.11 \%$ \\
\hline $\begin{array}{c}\text { Métodos de análise em química: caracterização e quantificação + cinética química (química geral) + } \\
\text { Compostos orgânicos: reações e mecanismos; macromoléculas naturais e sintéticas + A } \\
\text { experimentação no ensino de Química }\end{array}$ & 1 & $1.11 \%$ \\
\hline $\begin{array}{l}\text { Compostos orgânicos: reações e mecanismos; macromoléculas naturais e sintéticas + Bioquímica: } \\
\text { estrutura de biomoléculas, biossíntese e metabolismo + A experimentação no ensino de Química + } \\
\text { Compostos orgânicos + bioquímica }\end{array}$ & 1 & $1.11 \%$ \\
\hline $\begin{array}{l}\text { Estratégias de ensino e de avaliação em Química e suas relações com as diferentes concepções de } \\
\text { ensino e aprendizagem + Recursos didáticos para o ensino de Química }\end{array}$ & 1 & $1.11 \%$ \\
\hline $\begin{array}{l}\text { Projetos e propostas curriculares no ensino de Química + Relações entre ciência, tecnologia, sociedade } \\
\qquad \text { e ambiente no ensino de Química }\end{array}$ & 1 & $1.11 \%$ \\
\hline Projetos e propostas curriculares no ensino de Química + Recursos didáticos para o ensino de Química & 1 & $1.11 \%$ \\
\hline $\begin{array}{l}\text { Estudo de substâncias e transformações químicas (físico-química) + Métodos de análise em química: } \\
\text { caracterização e quantificação + Termodi nâmica (química geral) }\end{array}$ & 1 & $1.11 \%$ \\
\hline
\end{tabular}

RC: 80986

Ссылка доступа: https://www.nucleodoconhecimento.com.br/образованиe-ru/матрицы- 


\begin{tabular}{|c|c|c|}
\hline $\begin{array}{c}\text { A experimentação no ensino de Química + termodinâmica (química ge ral) + Métodos de análise em } \\
\text { quími ca: caracterização e quantificação + Estudo de substâncias e transformações químicas (físico- } \\
\text { química) }\end{array}$ & 1 & $1.11 \%$ \\
\hline $\begin{array}{c}\text { Elementos químicos (química inorgânica) + Estrutura atômica (química ge ral) + Estrutura molecular } \\
\text { (química inorgânica) + Estudo de substâncias e transformações químicas (físico-química) + Métodos de } \\
\text { análise em quími ca: caracterização e quanti ficação + Eletroquímica (química geral) + Estratégias de } \\
\text { ensino e de avaliação em Química e suas relações com as diferentes concepções de ensino e } \\
\text { aprendizagem + Estrutura molecular e de sólidos iônicos e metálicos }\end{array}$ & 1 & $1.11 \%$ \\
\hline $\begin{array}{c}\text { Projetos e propostas curriculares no ensino de Química + Estratégias de ensino e de avaliação em } \\
\text { Química e suas relações comas diferentes concepções de ensino e aprendizagem + Recursos didáticos } \\
\text { para o ensino de Química }\end{array}$ & 3 & $3.33 \%$ \\
\hline
\end{tabular}

Die Inhalte, die die längste Zeit innerhalb der Matrix des Bachelor-Studiengangs in Chemie des Instituto Federal de Educação, Ciência e Tecnologia do Amapá (IFAP), Macapa Campus haben, unter Berücksichtigung der 400 Stunden im Raum jeder Periode, ist Betreutes Praktikum in Lehre in Chemie I. Dieser Kurs hat 160 Stunden und stellt $40 \%$ der Gesamtstunden der 6 . Periode und 6,67\% der Gesamtstunden des Kurses dar.

Als nächstes erscheinen Grundlagen der Chemie und sozio-historische Grundlagen der Bildung (1. Periode); Philosophie der Erziehung und Berufsethik (2. Periode); Psychologie der Erziehung (3. Periode); Allgemeine Didaktik (4. Periode); Quantitative Analytische Chemie und Gesetzgebung und öffentliche Politik (5. Periode). Jede davon entspricht 80 Stunden Stunden, 20\% der Gesamtstunden ihrer jeweiligen Perioden und 3,33\% der Gesamtstunden des Kurses.

Inhalte wie Methodik der wissenschaftlichen Arbeit und Kommunikation und Sprache (1. Zeitraum); Allgemeine Chemie I, Physik I, Differential- und Integralrechnung I und Lineare Algebra und Analytische Geometrie (2. Periode); Allgemeine Chemie II, Physikalische Chemie I, Physik II und Differential- und Integralrechnung II (3. Periode); Physikalische Chemie II, Anorganische Chemie I; Organische Chemie I; und Qualitative Analytische Chemie (4. Periode); Anorganische Chemie II und Organische Chemie II (5. Periode) machen 60 Arbeitsstunden, 15\% der Gesamtstunden ihrer jeweiligen Perioden und 2,50\% der Gesamtstunden des Kurses aus.

RC: 80986

Ссылка доступа: https://www.nucleodoconhecimento.com.br/образование-ru/матрицы- 
Die anderen Disziplinen (Inhalte) machen 40 Arbeitsstunden, $10 \%$ der Gesamtstunden ihrer jeweiligen Perioden und 1,67 \% der Gesamtstunden des Kurses aus (Tabelle 2).

Tabelle 2 - zeigt die Verteilung der Disziplinen (Inhalte) des Bachelor-Studiengangs Chemie des Bundesinstituts für Bildung, Wissenschaft und Technologie von Amapa (IFAP), Macapa Campus. Jeder Inhalt zeigt seine Arbeitslast, den Prozentsatz innerhalb des Zeitraums und seinen Prozentsatz, der sich auf den gesamten Kurs bezieht.

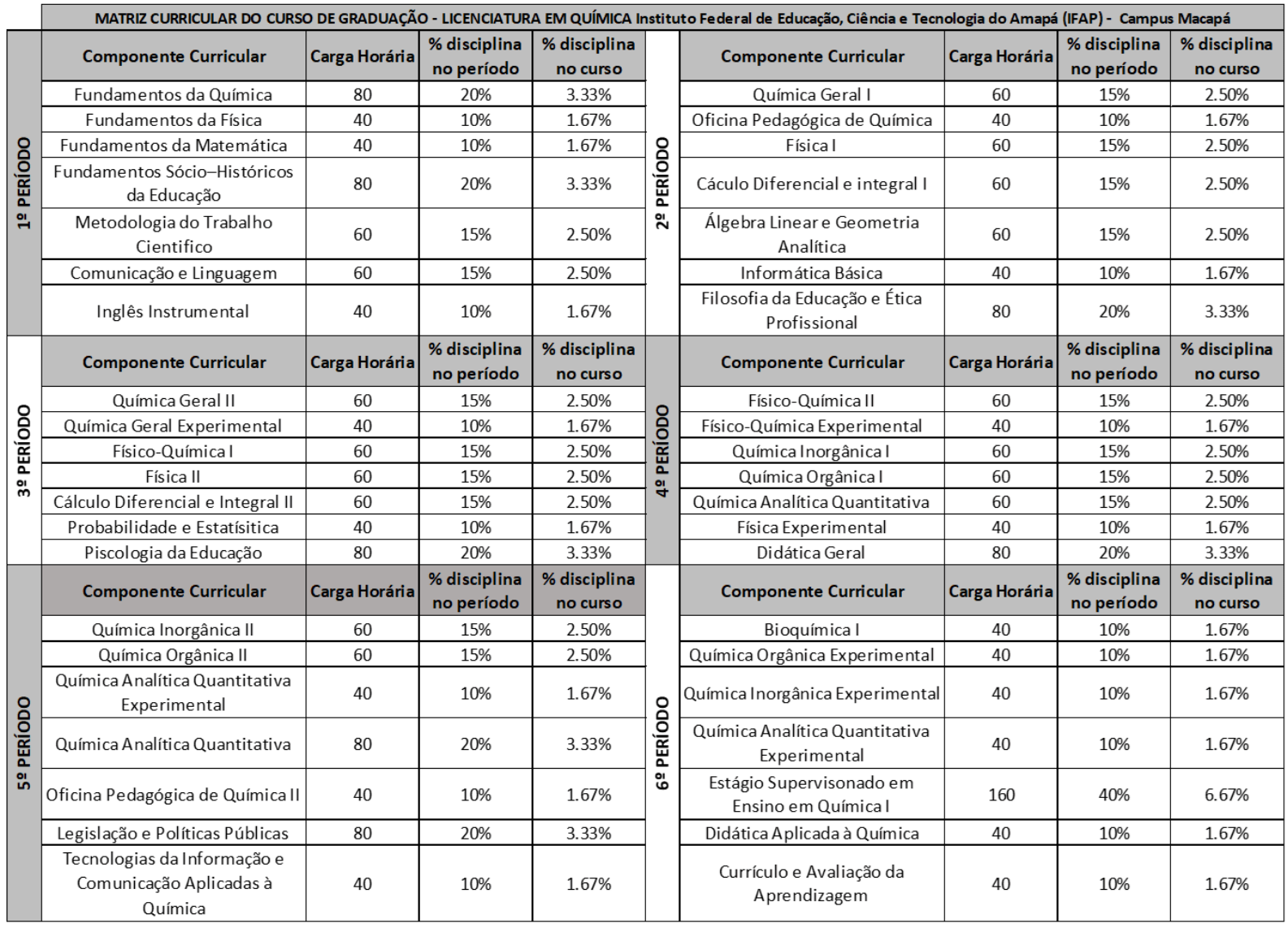

\section{DISKUSSION}

In den in Abbildung 1 dargestellten Daten kann eine größere Präferenz für Themen festgestellt werden, die Interdisziplinarität erfordern, da diese Fragen Wissen erfordern, das unterschiedliche Inhalte integriert, die gelöst werden müssen. Dies wird RC: 80986

Ссылка доступа: https://www.nucleodoconhecimento.com.br/образование-ru/матрицы- 
wichtig, weil die Fähigkeit, verschiedene Themen zu verbinden scheint ein solideres Lernen zu gewährleisten, und hilft gleichzeitig, die wissenschaftlichen Kenntnisse des Individuums aufzubauen. Die Prüfung verlangt, dass der Akademiker sich dem individuellen Wissen jedes Fachs anschließt und sich gleichzeitig mit anderen Disziplinen (SOUSA et al., 2017). Die Interdisziplinarität der Fragen stellt sicher, dass der Akademiker die Probleme interpretiert, die sie mit dem täglichen Leben verbinden, und ermöglicht die Wechselbeziehung von Wissen in verschiedenen Bereichen (Coelho e Scremin, 2019; Carmo et al., 2021; Gortz et al., 2021).

ENADE ist besorgt, dass der Student, der einen höheren Kurs in Chemie absolviert hat, die Gesetze versteht, die den Unterricht in dieser Disziplin regeln, und wie sich diese Normen auf diese Lehre auswirken (Tabelle 1). Die Ausbildung eines Fachkundigen, der kritisch und kenntnisreich über die Normen und die pädagogische Realität, von denen er tätig ist, kann dazu führen, dass dieser Lehrer zu signifikanten Verbesserungen der Lehrmethoden der Chemie beiträgt, was dazu führt, dass die Wissensrate zusammen mit der Verbesserung des Lebens der Schüler zunimmt (Pontes et al., 2008).

Die andere Sorge, die in den analysierten Tests bestand, war die organische Zusammensetzung von Lebewesen (Tabelle 1). Chemische Reaktionen lassen Zellen funktionieren, wenn also ein Chemielehrer die zelluläre Funktion kennt, kann er die Funktion organischer Moleküle näher an den Alltag der Schüler anführen (Leite und Velani, 2019). Die Inhaltlichkeit in den Blick des Tages der Studierenden zu stellen, hilft, diese Moleküle und ihre Prozesse zu verstehen (Hipólito e Silveira, 2011; Silveira et al., 2014).

Auch in Tabelle 1 sind 3,33\% der sich überschneidenden Fragen mit dem Wissen über Projektgestaltung, Lehrstrategien und Evaluation in der Chemie verbunden. Die Berufsausbildung in der Hochschulbildung im Bachelor-Studium neigt dazu, akademisches Wissen mit anderen zu integrieren, die speziell auf den Inhalt der Chemie zugeschnitten sind. Die Teilnahme von Lehrkräften an der Ausbildung, noch während der Hochschulbildung, an betreuten Praktika und an wissenschaftlichen RC: 80986

Ссылка доступа: https://www.nucleodoconhecimento.com.br/образование-ru/матрицы- 
Initiationsprogrammen, die vom Verband gefördert werden, scheint einen wesentlichen Beitrag zu ihren kritischen und akademischen Inhalten zu leisten. Dies würde die in diesen Fragen berechneten Eigenschaften verbessern (Rosa et al., 2018).

Die Daten zeigen, dass die wichtigsten in ENDADE in diesem Zeitraum gesammelten Inhalte öffentliche Politik, organische Zusammensetzung von Lebewesen, Projektgestaltung, Lehrstrategien und Evaluation in der Chemie waren (Tabelle 1). Die Curriculummatrix des Chemiestudiums am IFAP scheint dieses Wissen zu stützen (Tabelle 2). Beaufsichtigte Praktika, allgemeine Didaktik und Gesetzgebung und öffentliche Politik entsprechen 13\% (400 Stunden) der Gesamtstunden des Kurses. Organische Chemie I und II haben 160 Stunden zusammen und machen 5\% der gesamten Kursstunden aus. So steht der Pädagogische Studienplan (PPC), wie vom Kollegium der Hochschule, in der er sich befindet, für sich in Einklang hält, im Einklang mit den Änderungen der Bundesgesetzgebung für die Ausbildung von Lehrern in Bachelor-Studiengängen. Der analysierte Kurs respektiert Regionalitäten und dies spiegelt sich in der Art der Ausbildung seiner Studenten wider (Santos et al., 2020).

\section{SCHLUSSFOLGERUNG}

Die Bewertung von ENADE für Chemie-Absolventen erfordert, dass der ausgebildete Fachmann wie erwartet über Kenntnisse über spezifische Inhalte (z.B. organische Chemie) verfügt. In innen wird jedoch ein Wissen gesucht, das die Dynamik eines Klassenzimmers umfasst (in Bezug auf die Art der Klasse, Bewertung, wie man an eine Curriculummatrix denkt, wie man zu Veränderungen beiträgt, um den Unterricht zu verbessern, wie man einen Inhalt bewertet). Auf dieser Grundlage gibt es eine Vorliebe für bestimmte und unspezifische Fächer, die Informationen über die praktische Ausbildung der Studenten suchen.

Der Studiengang Chemie des Bundesinstituts für Amapá, IFAP, scheint mehr als genug Arbeitsbelastung zu haben, so dass der Student sich in ENADE gut vorbereiten und gut abschneiden kann, denn es folgt mit Verbesserungen, was den Bundesstandard für diese Art von Kurs regelt.

RC: 80986

Ссылка доступа: https://www.nucleodoconhecimento.com.br/образование-ru/матрицы- 


\section{VERWEISE}

CARMO, D. R. D. et al. A física no enem e no curso técnico de química do instituto federal do amapá (IFAP): Uma comparação curricular. Revista Científica Multidisciplinar Núcleo do Conhecimento, v. 3, p. 80-88, 2021. Disponível em: < https://www.nucleodoconhecimento.com.br/educacao/fisica-no-enem >.

CARNEIRO, J. D. et al. Matriz Curricular para Cursos de Ciências Contábeis. Brasilia DF: Fundação Brasileira de Contabilidade, 2017. 208p.

COELHO, F. B. O.; SCREMIN, G. A Interdisciplinaridade nas Licenciaturas em Ciências da Natureza: Análise de Projetos Pedagógicos de Cursos. XII Encontro Nacional de Pesquisa em Educação em Ciências - XII ENPEC Natal, RN: Universidade Federal do Rio Grande do Norte 2019.

ENADE. Antes da Prova, 2020. Brasilia DF, 2020. Disponível em: < https://www.gov.br/inep/pt-br/acesso-a-informacao/perguntas-frequentes/examenacional-de-desempenho-dos-estudantes-enade >. Acesso em: 02 fev 2021.

GORTZ, J. S. et al. Química do ensino médio técnico e enem: Uma comparação curricular. Revista Científica Multidisciplinar Núcleo do Conhecimento, v. 3, p. 8999 , 2021.

Disponível em:

https://www.nucleodoconhecimento.com.br/educacao/comparacao-curricular >.

HIPÓLITO, A. F.; SILVEIRA, H. E. D. As questões de Química do Exame Nacional do Ensino Médio (ENEM) em um enfoque transversal e interdisciplinar. 2011. Disponível em: < http://abrapecnet.org.br/atas_enpec/viiienpec/resumos/R0237-1.pdf >. Acesso em: 11 fev 2021.

IFAP. PPC Licenciatura em Química. Macapá AP, 2011. Disponível em: < https://macapa.ifap.edu.br/index.php/mais-noticias/395-licenciatura-em-quimica >. Acesso em: 02 fev 2021.

RC: 80986

Ссылка доступа: https://www.nucleodoconhecimento.com.br/образование-ru/матрицыбакалавриата 
INEP. Provas e Gabaritos ENADE, 2020. Brasilia DF, 2020. Disponível em: < http://inep.gov.br/educacao-superior/enade/provas-e-gabaritos >. Acesso em: 14 jul 2020.

LEITE, K. D. C.; VELANI, V. Divertindo-se com a química: o ensino e a aprendizagem por meio do lúdico. Braz. J. of Develop., v. 5, n. 11, p. 25115-25133, 2019.

LIMA, P. D. S. N. et al. Análise de dados do Enade e Enem: uma revisão sistemática da literatura. Avaliação (Campinas), v. 24, n. 1, p. 89-107, 2019.

PONTES, A. N. et al. O Ensino de Química no Nível Médio: Um Olhar a Respeito da Motivação. XIV Encontro Nacional de Ensino de Química (XIV ENEQ) Curitiba PR: Universidade Federal do Paraná 2008.

ROSA, D. L.; MENDES, A. N. F.; LOCATELLI, A. B. A formação da identidade docente na licenciatura em química e suas relações com a aprendizagem significativa a partir da análise do Modelo de Ensino de Gowin. Revista Práxis, v. 10, n. 20, dez., 2018, v. $10, \quad$ n. $20, \quad$ p. 1-14, 2018. Disponível em: < http://revistas.unifoa.edu.br/index.php/praxis/article/view/830/2237 >.

SANTOS, D. R. C. M. D.; LIMA, L. P.; JUNIOR, G. G. A formação de professores de química, mudanças na regulamentação e os impactos na estrutura em cursos de licenciatura em química. Quim. Nova, v. 43, n. 7, p. 977-986, 2020. Disponível em: $<$ https://www.scielo.br/pdf/qn/v43n7/0100-4042-qn-43-07-0977.pdf >.

SILVEIRA, F. L.; STILCK, J.; BARBOSA, M. Comunicações: Manifesto sobre a qualidade das questões de Física na Prova de Ciências da natureza no Exame Nacional de Ensino Médio. Caderno Brasileiro de Ensino de Física, v. 31, n. 2, p. 473-479, 2014.

WAINER, J.; MELGUIZO, T. Políticas de inclusão no ensino superior: avaliação do desempenho dos alunos baseado no Enade de 2012 a 2014. Educ. Pesqui., v. 44, p. e162807, 2018.

RC: 80986

Ссылка доступа: https://www.nucleodoconhecimento.com.br/образование-ru/матрицы- 
Eingereicht: März, 2021.

Genehmigt: März 2021.

RC: 80986

Ссылка доступа: https://www.nucleodoconhecimento.com.br/образование-ru/матрицыбакалавриата 